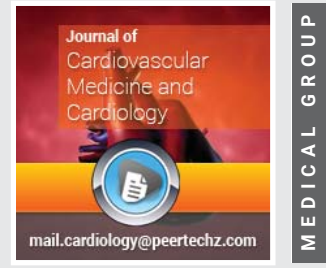

\title{
Diagnosis and management of a rare case of ruptured sinus of Valsalva into the right atrium
}

\author{
Charan Reddy KV ${ }^{1 *}$, Bhavesh Vajifdar ${ }^{1}$, Pavan Kumar ${ }^{2}$ and \\ Aniket Vazirani ${ }^{2}$ \\ ${ }^{1}$ Department of Clinical and Interventional Cardiology, Lilavati Hospital \& Research Centre, Bandra \\ (West), Mumbai-50, India \\ 2Department of Cardiovascular and Thoracic surgery, Lilavati Hospital and Research Center, Mumbai, \\ India
}

Received: 04 February, 2019
Accepted: 18 February, 2020
Published: 19 February, 2020

*Corresponding author: Dr. Charan Reddy KV, Department of Clinical and Interventional Cardiology, Lilavati Hospital \& Research Centre, Bandra (West), Mumbai-50, India, E-mail: chrnr@rediffmail.com

Keywords: Valsalva Aneurysm; Echocardiography; Coronary Sinus; Right Atrium

https://www.peertechz.com

Check for updates

\begin{abstract}
Sinus of Valsalva Aneurysm (SOVA) can arise from any of the three aortic sinuses. Aneurysm arising from the right coronary sinus, rupturing into the right atrium is extremely uncommon. SOVA's are seen more in males than females and in Asians than other ethnic groups. Majority of the cases are diagnosed during routine echocardiography and conventional angiography. However, with the availability of other advanced imaging techniques, such as cardiac Computed Tomography (CT) and Magnetic Resonance (MR) has not only improved the diagnosis but also its treatment. We report the case of a SOVA affecting the right coronary sinus and its subsequent management.
\end{abstract}

\section{Introduction}

Ruptured Sinus of Valsalva Aneurysm (RSOVA) is a rare lesion seen in both pediatric and adult population [1-3]. A right sinus of Valsalva aneurysm usually ruptures into the right ventricle and the aneurysms of non-coronary sinus into the right atrium [4]. Most sinus of Valsalva aneurysms are diagnosed on the basis of echocardiography, with or without angiography [5]. Here we present a rare case of a right sinus of Valsalva aneurysm, which ruptures into the right atrium.

\section{Case report}

A 36-year-old male with no known co-morbidities, presented with chief complaints of palpitation and progressive dyspnoea on exertion (NYHA class II) since the last 3months. He gives no history of alcohol, smoking, tobacco consumption or any cardiac interventions in the past. On examination, his heart rate was $82 / \mathrm{min}$, blood pressure was $120 / 30 \mathrm{~mm} \mathrm{Hg}$. The pulse was bounding with "water hammer character". Jugular Venous Pressure (JVP) was elevated (7cm above sternal angle) with prominent A wave. Arm span to height ratio was $<1$.
On auscultation of chest, S1 was normal and S2 was normally split with loud P2 component. There was a high-pitched, grade $4 / 6$, continuous murmur with maximum intensity in systole at the lower left sternal edge, with intensity of murmur increasing in sitting forward position and on clenching of fist.

Electrocardiogram (ECG) showed normal sinus rhythm with QRS axis of $+60^{\circ}$. X ray of chest revealed enlargement of mediastinum and cardiomegaly with right atrial enlargement dilated right and left pulmonary arteries with pulmonary plethora.

2D-Echocardiographic (2D-ECHO) images showed a large aneurysm at sinus of Valsalva arising from the right coronary cusp and protruding into right atrium with continuous turbulent flow into right atrium. No other cardiac abnormality was detected (Figure 1a,b). Aortogram, coronary angiography and cardiac catheterization was done. Aortogram showed a large saccular aneurysm of the right coronary sinus with contrast extravasation into the low pressure chamber of right atrium (Figure 2). CAG showed a right dominant circulation with ectasia of right coronary artery but without any coronary artery 

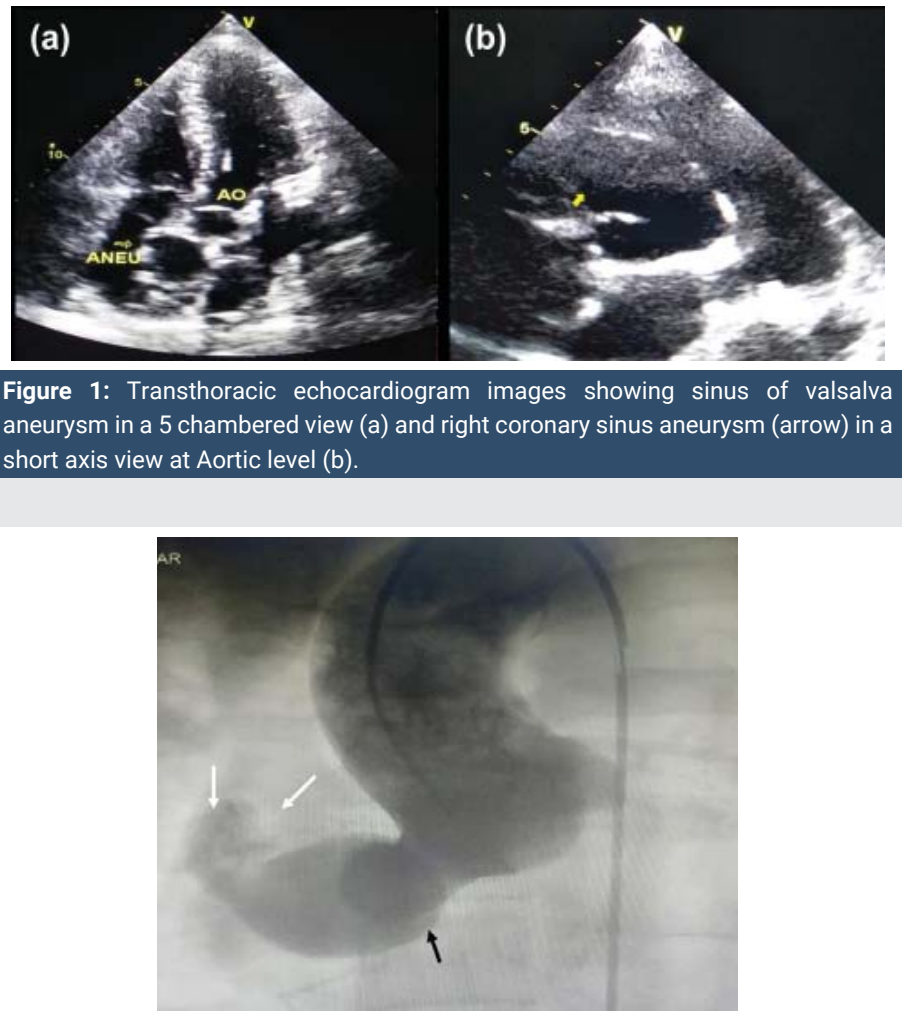

Figure 2: Aortic root angiographic film showing aneurysm of right sinus of valsalva (black arrow) with contrast extravasion into the RA chamber (white arrows).

disease or anatomical variation of the coronary circulation. There was a step up in oxygen saturation of the right atrium $(88 \%)$ compared to IVC $(68 \%)$ and SVC $(72 \%)$ on right heart catheterization confirming a left to right shunt.

The patient was initially managed with diuretics and vasodilators followed by surgical repair of RSOV aneurysm. Percutaneous device closure was not attempted due to insufficient margins of the RSOV and its proximity to the aortic valve.

\section{Surgical technique}

After establishing routine Cardiopulmonary Bypass (CPB), Right Coronary Sinus (RCS) aneurysm and its track identified. This track was found to be entering the inter-atrial septum with a cherry sized aneurysmal sac bulging into Right Atrium (RA). Surgery involved exposing the full fistulous track with excision of the redundant sac in the RA. The fistulous opening in RA to aortic sinus was closed with a patch. This was followed by reconstruction of inter-atrial septum (Figure 3a,b). Rest of the surgery involved termination of $\mathrm{CPB}$ and routine surgical closure. Intra-operative Transesophageal Echocardiography (TEE) showed closure of fistulous track with no residual left to right shunt. He made an uneventful recovery and was discharged seven days after surgery.

\section{Discussion}

SOVA was first described by John Thurman in year 1840 [6]. SOVA's are thin-walled outpouchings from the sinus which are usually tubular or saccular in shape. They are formed as the result of separation between aortic media and annulus fibrosis

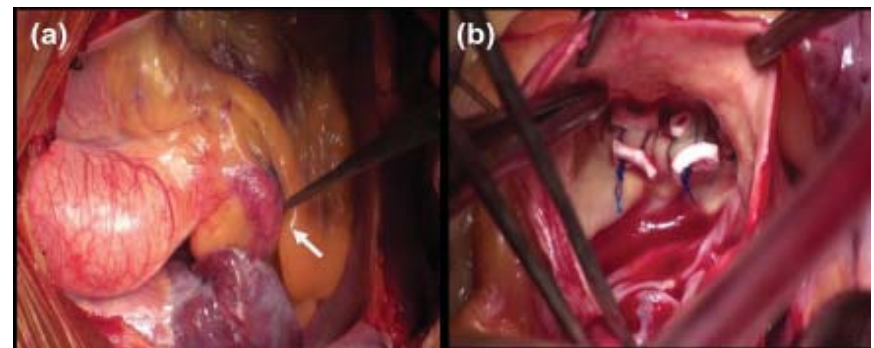

Figure 3: Intra-operative image of right coronary sinus aneurysm (arrow) arising from the base of aorta (a), and subsequent patch repair of the fistulous opening of right coronary sinus of valsalva into the right atrium (b)

[7], weakening the aortic root causing aneurysms. They are commonly associated with Marfan's syndrome, syphilis, and infective endocarditis of aortic valve. Acquired causes also include surgery to aortic root or valve and during coronary interventions [8].

RSOVs account for $1 \%$ of congenital anomalies of the heart and usually involve a single sinus and very rarely more than one sinus is involved. SOVA originates most commonly from the right coronary sinus $(70 \%-90 \%)$, followed by noncoronary sinus $(10 \%-20 \%)$ and rarely from left sinus $(<5 \%)$ [9]. Approximately, $50 \%$ of individuals with SOVA also have associated Ventricular Septal Defect (VSD) [10]. Bicuspid aortic valve, Coarctation of aorta (CoAo), pulmonary stenosis, and atrial septal defect are less commonly associated anomalies. About $10 \%$ of patients with Marfan's syndrome have SOVA.

Unruptured aneurysms are usually asymptomatic. They are incidentally detected on 2D-echocardiography. Rupture typically occurs in young males after puberty with male-tofemale ratio of 4:1. RSOV is five times more common in Asians as compared to Western population.

Aneurysm from right coronary sinus usually rupture into Right Ventricle (RV) and non coronary sinus rupture into RA. Rupture of left coronary sinus is rare and may cause pericardial effusion. Aneurysms of right sinus that rupture present with acute-onset chest pain that resolves after some time but is soon followed by right-sided heart failure symptoms which are progressive in nature. However, only $30 \%$ of the patients present with chest pain or severe dyspnea of acute onset, and the rest usually complain of progressive dyspnoea worsening with time. Death from Heart Failure (HF) typically occurs within a year after rupture. Hence, all the RSOV aneurysms should be repaired either percutaneously or surgically on detection. Large aneurysms can also compress the coronary arteries causing angina as a predominant symptom.

Diagnosis of RSOV is often established by history and clinical examination. ECG may show evidence of chamber enlargement and features of volume overload of heart. Chest $\mathrm{X}$-ray may also show cardiomegaly with increased pulmonary vascularity. Blood cultures should be taken at admission to rule out active infective endocarditis. Transthoracic Twodimensional Echo (TTE) showing typical "windsock deformity" clinches the diagnosis. Transoesophageal ECHO (TEE) allows identification of other associated structural abnormalities. A definitive diagnosis can be made with aortography and cardiac 
catheterization. Noninvasive techniques such as cardiac Computed Tomography (CT) and Magnetic Resonance Imaging (MRI) may also be used to delineate the RSOV and associated cardiac anomalies.

RSOV aneurysms need definitive therapy. Patients require initial stabilization and control of heart failure symptoms with diuretics, vasodilators and inotropes. Treatment options include surgical repair or percutaneous device closure. The factors determining the choice of treatment includes anatomic location of the RSOV and associated cardiac defects. Surgical repair is still the gold standard in the management of RSOV with in-hospital mortality of less than $5 \%$. It is usually indicated in patients who have associated VSD or large RSOV with aortic end $>12 \mathrm{~mm}$, RSOV with multiple shunts or suspicion or evidence of aortic valve endocarditis.

Percutaneous closure of RSOV was first attempted by Cullen, et al., in 1994 using a Rashkind umbrella [11]. Catheter closure of RSOV is now a safe and feasible alternative to surgical repair in patients with favourable anatomy without associated cardiac lesions. Most commonly used occlusion devices are Amplatzer Duct Occluder (ADO), Amplatzer Septal Occlude (ASO) and muscular VSD closure device. Complications of catheter closure include device migration, hemolysis, and encroachment on to aortic valve leaflets or coronary ostia and AV conduction disturbance.

\section{Conclusion}

Rupture of right sinus of Valsalva into RA is a rare occurrence. Early detection and management is vital to reduce morbidity and mortality.Tetrad of features such as continuous murmur, elevated pulsating JVP, bounding pulse along with a history of sudden-onset chest or epigastric pain is highly suggestive of RSOV aneurysm [12]. Imaging techniques play a pivotal role in early diagnosis and treatment. Treatment options include surgical repair or percutaneous device closure based on anatomy and associated cardiac defects.

\section{Acknowledgement}

The authors are thankful to the patient for consenting to publish this case report. We are also thankful to Dr. Vidya Suratkal and her staff, Department of Cardiology, Lilavati Hospital and Research Centre, Mumbai for their support during the study.

\section{References}

1. Fazio G, Zito R, Dioco DD, Mussagy C, Loredana S, et al. (2006) Rupture of a left sinus of Valsalva aneurysm into the pulmonary artery. European $\mathrm{J}$ Echocardiography 7: 230-232. Link: http://bit.ly/2P2uYrC

2. Weinreich M, Yu PJ, Trost B (2015) Sinus of Valsalva Aneurysms: Review of the Literature and an Update on Management. Clin Cardiol 38: 185-189. Link: http://bit.ly/2V3bx5R
3. Jain A, Achuthan G (2019) Rupture of Sinus of Valsalva Aneurysm into Inter-ventricular Septum: Role of Cardiac CT. Cureus 11: e5589. Link: http://bit.ly/2V619dA

4. Mayer ED, Ruffmann K, Saggau W (1986) Ruptured aneurysms of the sinus of Valsalva. Ann Thorac Surg 42: 81-85. Link: http://bit.ly/3bQ6RpW

5. Bricker AO, Avutu B, Mohammed TL (2010) Valsalva sinus aneurysms: findings at CT and MR imaging. Radiographics 30: 99-110. Link: http://bit.ly/37AL3et

6. Thurman J (1840) On aneurisms, and especially spontaneous varicose aneurisms of the ascending aorta, and sinuses of Valsalva: with cases. Med Chir Tr 23: 323-384. Link: http://bit.ly/2HxYhhL

7. Edwards JE, Burchell HB (1957) The pathological anatomy of deficiencies between the aortic root and the heart, including aortic sinus aneurysms. Thorax 12: 125-139. Link: http://bit.ly/2SW3eWN

8. Zanjani KS, Niwa K (2013) Aortic dilatation and aortopathy in congenital heart diseases. J Cardiol 61: 16-21. Link: http://bit.ly/3blzVj0

9. Hoey ET, Kanagasingam A, Sivananthan MU (2010) Sinus of valsalva aneurysms: Assessment with cardiovascular MRI. AJR Am J Roentgenol 194 495-504. Link: http://bit.ly/2SPEGyw

10. Csu SH, Hung CR, How SS, Chang H, Wang SS, et al (1990) Ruptured aneurysms of the sinus of Valsalva in oriental patients. J Thorac Cardiovasc Surg 99: 288 298. Link: http://bit.ly/2VeXp9V

11. Cullen S, Somerville J, Redington A (1994) Transcatheter closure of ruptured aneurysm of the sinus of Valsalva. Br Heart J 71: 479-480. Link: http://bit.ly/2vQ32jR

12. Parashar NK, Bhasin D, Marotrao PSK (2017) Ruptured sinus of Valsalva aneurysm: Clinical case presentation and management. Curriculum in Cardiology-case discussion. 3: 109-114. Link: http://bit.ly/2wrWfxbs

Discover a bigger Impact and Visibility of your article publication with

\section{Peertechz Publications}

\section{Highlights}

* Signatory publisher of ORCID

* Signatory Publisher of DORA (San Francisco Declaration on Research Assessment)

* Articles archived in worlds' renowned service providers such as Portico, CNKI, AGRIS, TDNet, Base (Bielefeld University Library), CrossRef, Scilit, J-Gate etc.

* Journals indexed in ICMJE, SHERPA/ROMEO, Google Scholar etc.

* OAI-PMH (Open Archives Initiative Protocol for Metadata Harvesting)

* Dedicated Editorial Board for every journal

* Accurate and rapid peer-review proces

* Increased citations of published articles through promotions

* Reduced timeline for article publication

Submit your articles and experience a new surge in publication services (https://wWw.peertechz.com/submission).

Peertechz journals wishes everlasting success in your every endeavours.

Copyright: (๑ 2020 Charan Reddy KV, et al. This is an open-access article distributed under the terms of the Creative Commons Attribution License, which permits unrestricted use, distribution, and reproduction in any medium, provided the original author and source are credited.

Citation: Charan Reddy KV, Vajifdar B, Kumar P, Vazirani A(2020) Diagnosis and management of a rare case of ruptured sinus of Valsalva into the right atrium. $J$ Cardiovasc Med Cardiol 7(1): 033-035. DOl: https://dx.doi.org/10.17352/2455-2976.000108 